\title{
Programa de remediação fonológica para alunos com Síndrome de Down: aplicabilidade do método JT na Educação Especial
}

\author{
Roberta Moreno Sás* \\ Danila Secolim Coser** \\ Miriam Bratfisch Villa***

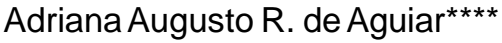 \\ Maria Amélia Almeida*****
}

\section{Resumo}

Analisar resultados de intervenções, verificando sua efetividade, constitui um desafio para a pesquisa e para a atuação profissional, principalmente quando os métodos tradicionais não são aplicáveis (por problemas de amostragem ou de dificuldade de replicação). Desta forma, torna-se necessário investir no desenvolvimento de métodos alternativos. Este artigo exemplifica a aplicação do "Método JT", método de análise da efetividade de intervenções, a partir do cálculo da Significância Clínica e do Índice de Mudança Confiável, para verificação dos efeitos de um programa de remediação fonológica realizado com oito alunos do ensino regular diagnosticados com Síndrome de Down. Os resultados da análise evidenciaram o potencial desse método para a avaliação da efetividade de programas de intervenção, tanto na análise de resultados individuais como em grupo. Discute-se a potencialidade do método para o aperfeiçoamento de programas de intervenção e encaminhamento para novas pesquisas.

Palavras-chave: Mudança confiável. Significância clínica. Tratamentos baseados em evidências.

\section{Phonological remediation program for students with Down Syndrome: aplicability of method JT in Special Education}

\section{Abstract}

Analyze results of interventions, assessing their effectiveness, is a challenge for research and professional performance, especially when traditional methods are not applicable, making it necessary development alternative methods. This article exemplifies the application of "Method JT", method for analyzing the effectiveness

\footnotetext{
* Fonoaudióloga, Mestre e Doutoranda em Educação Especial pela Universidade Federal de São Carlos. Departamento de Psicologia. São Calos, São Paulo, Brasil.

** Psicóloga, Mestre e Doutoranda em Educação Especial pela Universidade Federal de São Carlos. Departamento de Psicologia. São Calos, São Paulo, Brasil.

*** Psicóloga, mestre e doutora em Psicologia. Professora Voluntária do Departamento de Psicologia da UFSCar. Bolsista PRODOC/CAPES vinculada ao Programa de Pós Graduação em Educação Especial da UFSCar. São Calos, São Paulo, Brasil.

${ }^{\star \star \star \star}$ Fonoaudióloga, Mestre, Doutora em Educação Especial e Pós-Doutora em Psicologia das Habilidades Sociais pela Universidade Federal de São Carlos. Departamento de Psicologia. São Calos, São Paulo, Brasil.

***** Graduada em Letras, com mestrado, PhD e pós doutorado em Educação Especial. Professora associada da Universidade Federal de São Carlos, docente no Programa de Pós Graduação em Educação Especial da UFSCar. São Calos, São Paulo, Brasil.
} 
Roberta Moreno Sás - Danila Secolim Coser - Miriam Bratfisch Villa Adriana Augusto R. de Aguiar - Maria Amélia Almeida

of interventions, from the calculation of Clinical Significance and a Reliable Index Change to verify the interventions effects of a phonological remediation program carried out with eight students in regular education diagnosed with Down Syndrome. The analysis results showed the potential of this Method for the assessment the effectiveness of intervention programs, both the analysis of individual and group results. It discusses the potential of the method for improving intervention programs and referral for further research.

Keywords: Reliable change index. Clinical significance. Evidence-based treatments.

\section{Introdução}

A grande diversidade de pesquisas envolvendo intervenções clínicas na atualidade reabriu as discussões sobre a validade dos métodos estatísticos, comumente empregados nesses estudos, para análise da efetividade da intervenção. Há evidências de que uma mudança observada nos desempenhos de pré e pós-intervenção, calculada com base em estatística inferencial (paramétrica ou não paramétrica), pode ser considerada estatisticamente significativa e não ser clinicamente relevante, ou seja, que a existência de efeito do tratamento pode ter pouco a ver com o significado clínico desse efeito (JACOBSON; TRUAX, 1992). Usualmente a estatística é empregada para estimar a probabilidade de acaso envolvido na mudança ou ainda para estimar a magnitude das diferenças entre dois ou mais grupos comparados, sem contemplar as mudanças de indivíduos específicos dentro de cada grupo (OGLES, LAMBERT e MASTERS, 1996 apud YOSHIDA, 2008). Nessas situações faz-se necessário a utilização de procedimentos de avaliação de mudança entre o início e o final de uma intervenção, que apreendam significância clínica (ou seja, que tenham validade externa) e não apenas significância estatística.

Visto esse contexto, várias propostas surgiram para análise empírica da efetividade de intervenções (evidence-based pratices), principalmente no contexto da prática clínica. Um método estatístico para análise da efetividade de intervenção, que considera a confiabilidade da mudança e a significância clínica, foi desenvolvido inicialmente por Jacobson, Follette e Revenstorf (1984) e retomado posteriormente por Jacobson e Truax (1991) sendo conhecido atualmente por "Método JT" (DEL PRETTE; DEL PRETTE, 2008).

Para Jacobson e Truax (1992), a preocupação central era a de elaborar um procedimento de identificação de mudanças confiáveis e clinicamente significantes que fosse: (a) aplicável, pelo menos teoricamente, a qualquer desordem clínica; (b) consistente com as expectativas dos profissionais quanto aos resultados da psicoterapia; (c) útil na identificação dos clientes que se recuperaram, melhoraram e não melhoraram ou até pioraram após a intervenção. 
Como relatam Del Prette e Del Prette (2008, p. 497-498) e de acordo com Cozby (2003),

\begin{abstract}
"a efetividade de qualquer intervenção (clínica, educacional, organizacional etc.) implica, necessariamente, reunir evidências empíricas pertinentes sobre: (a) sua validade interna, ou seja, o quanto os resultados podem ser atribuídos aos procedimentos utilizados; e (b) sua validade social ou externa, em termos de impacto sobre o funcionamento adaptativo do cliente, generalização para outros ambientes ou para população mais ampla, aceitabilidade do tratamento, relação custo-benefício etc."
\end{abstract}

O Método JT se apóia em dois conceitos básicos: Significância Clínica (SC) e Índice Mudança Confiável (IMC). A SC está relacionada à validade externa, ou seja, o quanto a intervenção produziu mudanças efetivas do ponto de vista do participante, do clínico, da família e da sociedade em geral. O IMC serve para determinar se as mudanças verificadas na comparação entre a pré e a pós-intervenção podem ser atribuídas aos procedimentos utilizados ou à erros de medida, portanto, está relacionado à validade interna. Dessa forma, a análise da SC permite verificar o grau em que clientes atingiram os padrões esperados de melhora ou de recuperação, já o IMC serve para determinar se as mudanças entre a pré e a pós-intervenção verificadas para cada indivíduo ou para a média do grupo se devem aos procedimentos utilizados ou se constituem artefatos de medida (JACOBSON \& TRUAX,1992).

Para Del Prette e Del Prette (2008), o Método JT, além da aplicação como complemento à análise de significância estatística, representa uma alternativa para estudos nos quais a quantidade de sujeitos e/ou outros fatores (tais como a ausência de dados normativos e de grupo controle), inviabilizam a análise inferencial dos resultados com base em medidas de tendência central e dispersão. Contudo, ainda segundo tais autores, a potencialidade do método requer estudos empíricos sobre diferentes conjuntos de dados e problemas de pesquisas que poderiam contribuir para ampliar as informações de limitações e possibilidades deste.

Nessa perspectiva, e frente à necessidade de metodologias que avaliem as intervenções realizadas, principalmente nos contextos de clínicas e escolas, e considerando sua aplicabilidade em diversos setores, o Método JT vem sendo estudado e aplicado em análises de diversas intervenções abrangendo estudos das áreas de saúde e educação. No Brasil, ainda são poucos os estudos que utilizaram a metodologia JT, destacando-se o estudo de Yoshida (2008), que avaliou os processos de psicoterapia dinâmica breve; o de Pereira, Silvares e Del Prette (2008), que examinaram os efeitos do tratamento da enurese sobre a redução de problemas de comportamento e o de Aguiar (2006) e Aguiar et. al (2010), que efetuaram a análise de mudança confiável para os resultados 
Roberta Moreno Sás - Danila Secolim Coser - Miriam Bratfisch Villa Adriana Augusto R. de Aguiar - Maria Amélia Almeida

de um grupo de deficientes mentais adultos que participaram de um programa de promoção de habilidades sociais e comunicativas.

A fim de dar continuidade ao estudo da avaliação da efetividade e operacionalização do Método JT em diferentes contextos de intervenção, este artigo trás um exemplo de aplicação deste Método utilizando-se dos dados da Dissertação de Mestrado em Educação Especial da primeira autora, que teve, entre seus objetivos, a avaliação pré e pós-intervenção de habilidades de consciência fonológica de alunos com síndrome de Down submetidos a uma intervenção. Como aponta Aguiar et. al. (2010), algumas limitações da amostra e do delineamento do estudo, como o número reduzido de participantes e ausência de dados normativos, levaram à busca de procedimentos de análise de dados alternativos aos comumente utilizados. Tais limitações, que a princípio mostraram-se como obstáculos, ao longo do processo de estudo dos métodos de análise, revelaram a possibilidade da utilização do Método JT, com consequente contribuição para diferentes áreas que enfrentam situações de pesquisa e prática semelhantes, como é o caso da Educação Especial.

Este artigo tem como objetivo investigar a aplicabilidade do Método JT para avaliação de um programa de remediação fonológica para alunos com diagnóstico de Síndrome de Down. A intervenção baseou-se na remediação fonológica, que é o treino das habilidades de consciência fonológica. Há o consenso de autores como Capellini (2001); Capellini e Salgado (2003); Capellini, Padula e Ciasca (2004) e Salgado (2005), de que a criança, quando inicia a alfabetização, já possui domínio da linguagem oral, e assim possui as estruturas linguísticocognitivas preparadas para aprender a ler e escrever, porém ainda não relaciona a linguagem oral com os aspectos da leitura e da escrita. Em outras palavras, podemos dizer que apresentam dificuldades na consciência fonológica que de acordo com Bryant e Bradley (1987), pode ser entendida como um conjunto de habilidades que vão desde a simples percepção global do tamanho da palavra e de semelhanças fonológicas entre as palavras até a segmentação e manipulação de sílabas e fonemas. Tal conceito diz respeito tanto à habilidade de manipular esses segmentos quanto à compreensão de que a fala pode ser segmentada (CAPOVILLA; CAPOVILLA, 2004).

Ferreiro e Teberosky (1995) entendem que a aprendizagem da leitura necessita que a criança esteja atenta ao fato de que, a linguagem oral é composta de palavras e sentenças separadas, descobrindo que estas correspondem a unidades da fala. Além disso, a criança precisa se conscientizar sobre os fonemas. Portanto, esta consciência é imprescindível para a aprendizagem no sistema de escrita alfabético como o da Língua Portuguesa, pois geralmente as letras do alfabeto correspondem aos fonemas. 


\section{Método}

\section{Participantes}

Participaram do estudo oito (8) alunos com Diagnóstico da Síndrome de Down do tipo Trissomia do 21, matriculados em escola regular e enquadrados na fase alfabética, dentro da psicogênese da língua escrita, de acordo com a prova de quatro palavras e uma frase de Ferreiro e Teberosky (1995). Os participantes foram divididos aleatoriamente, por meio de sorteio, em dois grupos: 0 Grupo Experimental (GE) e o Grupo Controle de Espera (GCE). Os sujeitos do grupo experimental foram denominados GE1, GE2, GE3 e GE4 e do grupo controle de espera como GCE1, GCE2, GCE3 e GCE4.

O estudo foi realizado na escola onde os alunos estavam matriculados, em uma cidade de pequeno porte, com população de cerca de 21.000 habitantes (BRASIL - IBGE, 2007), localizada no interior do estado de São Paulo.

\section{Aspectos Éticos}

O Projeto de Pesquisa foi aprovado pelo Comitê de Ética em Pesquisa para Experimentos em Seres Humanos da Universidade Federal de São Carlos (CEP/UFSCar, Protocolo 270/2007).

\section{Instrumentos}

Para verificação da eficácia do programa de intervenção utilizou-se, antes e após a intervenção, a Prova de Consciência Fonológica (CAPOVILLA E CAPOVILLA, 2000), que consiste em dez subtestes, cada qual composto de quatro itens referentes às habilidades de síntese, segmentação, manipulação e transposição silábica e fonêmica, rima e aliteração. A prova permite um total de 40 pontos sendo quatro pontos de cada um dos dez subtestes. Por meio da Prova de Consciência fonológica pode-se avaliar a habilidade dos participantes do estudo de manipular sons da fala, expressando oralmente o resultado dessa manipulação.

\section{Procedimento de coleta de dados}

A intervenção seguiu o programa de remediação fonológica segundo González, Espinel e Rosquete (2002), adaptado para a população brasileira por Salgado (2005), apresentando oito etapas (correspondência grafema-fonema 1; discriminação de fonemas em sílabas; classificação de palavras pares; discriminação de fonemas em palavras; correspondência grafema-fonema 2; identificação de fonema; segmentação de fonema e supressão de fonemas). A intervenção foi realizada em sessões aplicadas três vezes por semana, com duração de 45 minutos e de forma individual.

O número de sessões e o período dispensado para o estudo variaram pouco entre os participantes do mesmo grupo e entre os dois grupos em questão. A Tabela 1 descreve os detalhes dessas variações por participante. 
Roberta Moreno Sás - Danila Secolim Coser - Miriam Bratfisch Villa Adriana Augusto R. de Aguiar - Maria Amélia Almeida

Tabela 1- Número de sessões realizadas com cada participante

\begin{tabular}{lcccccccccc}
\hline \multirow{2}{*}{ PARTICIPANTES } & $\begin{array}{c}\text { TOTAL } \\
\text { DE } \\
\text { SESSÕES }\end{array}$ & \multicolumn{1}{c}{ TOTAL DE SESSÕES EM CADA } \\
& & & $\mathbf{1}$ & $\mathbf{2}$ & $\mathbf{3}$ & $\mathbf{4}$ & $\mathbf{5}$ & $\mathbf{6}$ & $\mathbf{7}$ & $\mathbf{8}$ \\
\hline \multirow{4}{*}{$\begin{array}{l}\text { Grupo } \\
\text { Experimental }\end{array}$} & GE1 & 14 & 2 & 1 & 2 & 1 & 1 & 2 & 3 & 2 \\
& GE2 & 14 & 1 & 2 & 2 & 2 & 2 & 1 & 2 & 2 \\
& GE3 & 13 & 1 & 1 & 2 & 1 & 1 & 2 & 2 & 3 \\
Grupo & GE4 & 9 & 1 & 1 & 1 & 1 & 1 & 1 & 1 & 2 \\
Controle de & GCE2 & 15 & 2 & 1 & 2 & 1 & 1 & 2 & 3 & 3 \\
Espera & GCE3 & 13 & 1 & 1 & 2 & 2 & 1 & 2 & 2 & 2 \\
& GCE4 & 9 & 1 & 1 & 1 & 1 & 1 & 1 & 1 & 9 \\
\hline
\end{tabular}

* Número de sessões necessárias para atingir o total de acertos em cada Etapa

No grupo experimental, GE1 necessitou de 14 sessões, sendo que nas etapas 2, 4 e 5 atingiu os $100 \%$ de acertos em uma única sessão, nas etapas 1, 3, 6 e 8 precisou de duas sessões e, na etapa 7, três sessões foram necessárias para atingir o critério de $100 \%$ de acertos. O participante GE2 requereu 14 sessões, sendo uma sessão nas etapas 1 e 6 e duas nas demais etapas. GE3 necessitou 13 sessões, sendo distribuídas em uma sessão para as etapas $1,2,4,5$, duas sessões para as etapas 3, 6 e 7 e três sessões para a etapa 8. GE4 requereu nove sessões, sendo que a única em que não atingiu $100 \%$ de acertos na primeira sessão foi na etapa 8 e atingiu os $100 \%$ de acertos na etapa de intervenção com maior rapidez do que os demais participantes. Com exceção de GE1, cuja etapa de maior número de sessões necessárias foi a etapa 7, nos demais a etapa 8 foi a que mais requereu sessões. Assim, podese sugerir que foi a tarefa considerada mais difícil pelos participantes do GE. Em relação ao grupo controle de espera, o participante GCE1 demandou 13 sessões, sendo que nas etapas 1, 4 e 6 atingiu os $100 \%$ de acertos em uma única sessão e, nas demais etapas, precisou de duas sessões. GCE2 necessitou de um total de 15 sessões, sendo uma sessão para as etapas 2 , 4 e 5 , duas sessões para as etapas 1,3 e 6 e três sessões para as etapas 7 e 8 . Para GCE3, foram ao todo 13 sessões, sendo uma sessão para as etapas 1, 2 e 5 e duas sessões para as demais etapas. Já GCE4 necessitou de nove sessões, sendo que apenas na etapa 8 não atingiu 100\% de acertos na primeira sessão. 
Durante a intervenção, foi utilizado o delineamento de grupos. Inicialmente, os oito participantes, quatro do Grupo Experimental (GE) e o quatro do Grupo Controle de Espera (GCE) foram submetidos a um pré-teste. Em seguida, foi iniciada a intervenção com o GE. Após essa intervenção, o GE passou pelo pós-teste, enquanto que o GCE realizou o segundo pré-teste. Neste momento, teve início a intervenção com o GCE, que após o término da mesma, passou pelas provas de pós-teste.

\section{Análise dos dados}

Foi realizada a análise dos resultados por meio do Método JT, considerando o escore geral da Prova de Consciência Fonológica. Para os cálculos do Método JT, foram utilizadas a média e o desvio padrão baseando-se na amostra local (oito sujeitos que participaram deste estudo $n=08 ; M=16,5 ; D P=3,70$ ). A análise realizada por meio do Método JT esteve apoiada em seus dois conceitos centrais: IMC e a SC, os quais possuem equações específicas para o seu cálculo (Jacobson \& Truax, 1991, Aguiar, Aguiar \& Del Prette, 2009; Del Prette \& Del Prette, 2008; Villa et al, no prelo).

Para o cálculo do ponto de corte de significância clínica utilizou-se o Critério $A,{ }^{1}$ que segundo os autores, é utilizado quando não se dispõe de dados normativos da população funcional. Conforme descrito em Aguiar et. al. (2010) a análise dos dados a partir do Método JT pode ser feita obtendo-se uma representação gráfica das diferenças pré e pós-intervenção. Para compreensão dos resultados quanto à confiabilidade das mudanças deve-se observar a linha diagonal central que separa acima as diferenças positivas (pós>pré) e abaixo as negativas (pós<pré). Há ainda duas diagonais que delimitam uma área de incerteza, além da qual se pode afirmar que houve mudanças confiáveis. Quanto à significância clínica, a análise dos gráficos é realizada a partir dos quatro quadrantes formados pelo cruzamento da linha vertical e horizontal. As duas linhas serrilhadas representam a área de incerteza. Para afirmar ocorrência de mudança clinicamente significativa (na qual população clínica passou a ser não clínica) os participantes devem estar dispostos no quadrante acima das linhas horizontais e à esquerda das linhas verticais.

\section{Resultados}

Considerando o escore geral da Prova de Consciência Fonológica, no grupo experimental (GE), apresentado na Figura 1, houve mudança positiva confiável (MPC) para os participantes GE1, GE2 e GE3, enquanto o participante GE4 não apresentou mudança após a intervenção. Quanto à significância clínica é possível verificar que para dois participantes, GE1 e GE3, houve mudança clinicamente significativa. Dito de outra forma, os dados revelam que a intervenção proporcionou mudanças quanto a consciência fonológica para os sujeitos 1,2 e 3 do grupo experimental, sendo que tais mudanças foram suficientes para retirar os sujeitos 1 e 3 da população clínica (que necessita de intervenção). 


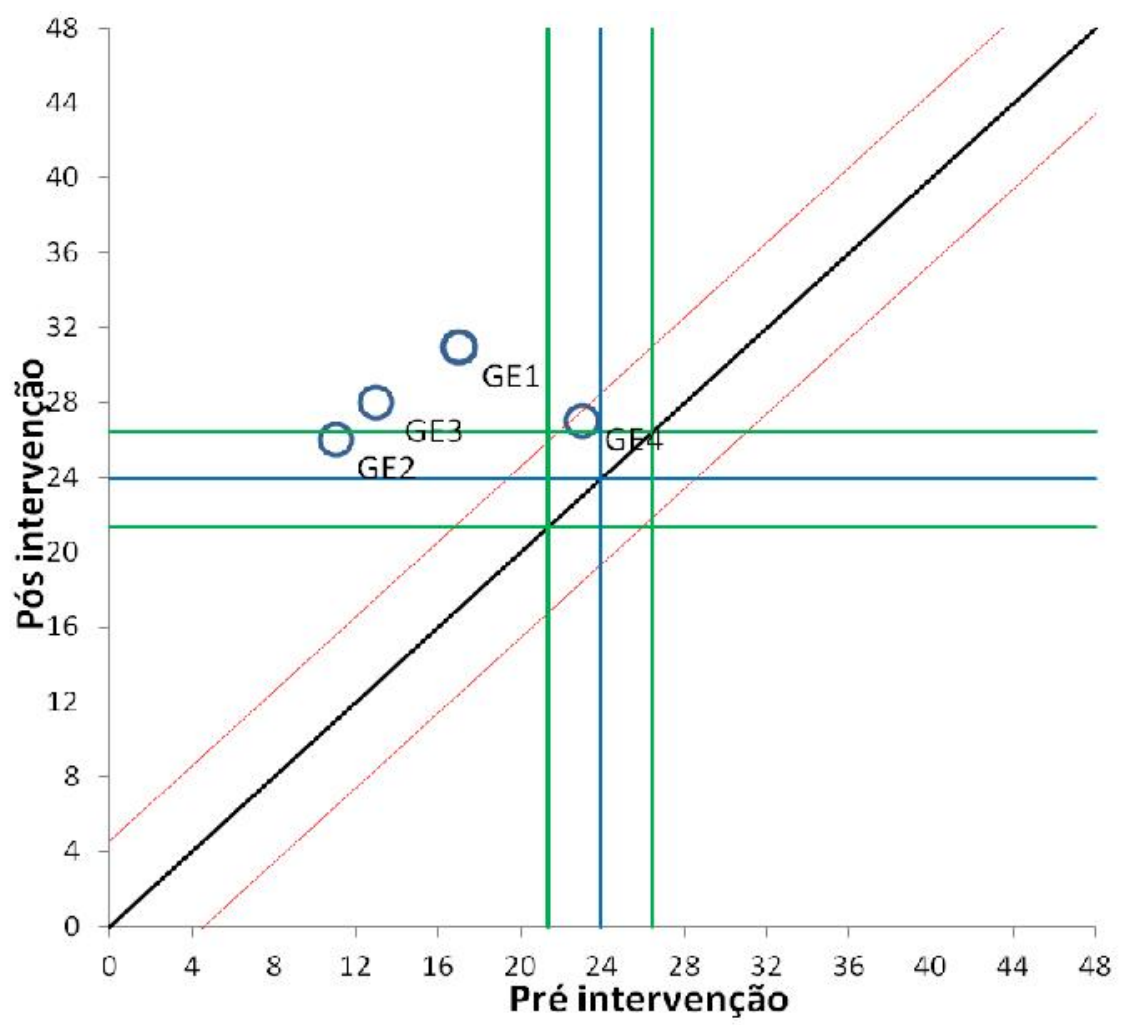

Figura 1 - Resultados da operacionalização do cálculo do IMC e da SC (Critério A) considerando o Escore Geral da Prova de Consciência Fonológica no Grupo Experimental nas situações de pré e pós-teste.

Quanto ao grupo Controle de Espera (GCE), a Figura 2 apresenta os resultados considerando o pré-teste 1 e pré-teste 2 , no qual ainda não havia sido feito a intervenção. É possível perceber, que nenhum participante apresentou mudança confiável, ou seja, a mera passagem de tempo ou outras variáveis da vida dos sujeitos não foram suficientes para mudanças significativas quanto à consciência fonológica. Também não houve mudança de status clínico no período correspondente ao pré-teste 1 e 2 , indicando que os sujeitos permaneceram na população clínica. 


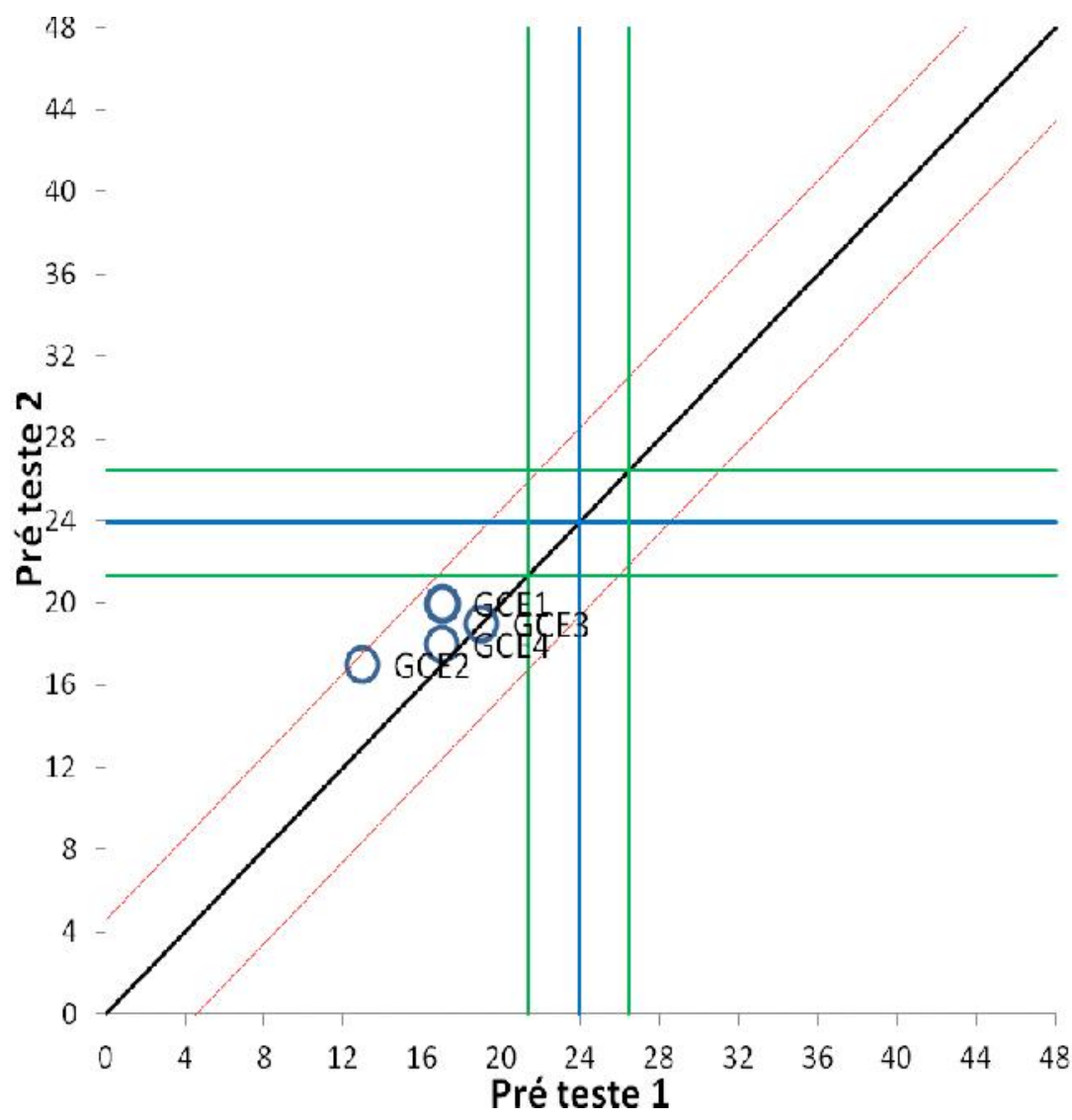

Figura 2 - Resultados da operacionalização do cálculo do ÍMC e da SC (Critério A) considerando o Escore Geral da Prova de Consciência Fonológica no Grupo Controle de Espera nas situações de pré-teste 1 e pré-teste 2.

A Figura 3 apresenta os dados considerando o grupo controle de espera (GCE) no período entre o pré-teste 1 e o pós-teste, realizado após a intervenção. Pode-se observar que para todos os participantes ocorreu uma mudança positiva confiável e que essa mudança pode ser considerada como clinicamente significativa, ou seja, que alcançou os critérios para ser considerada funcional para os envolvidos. 


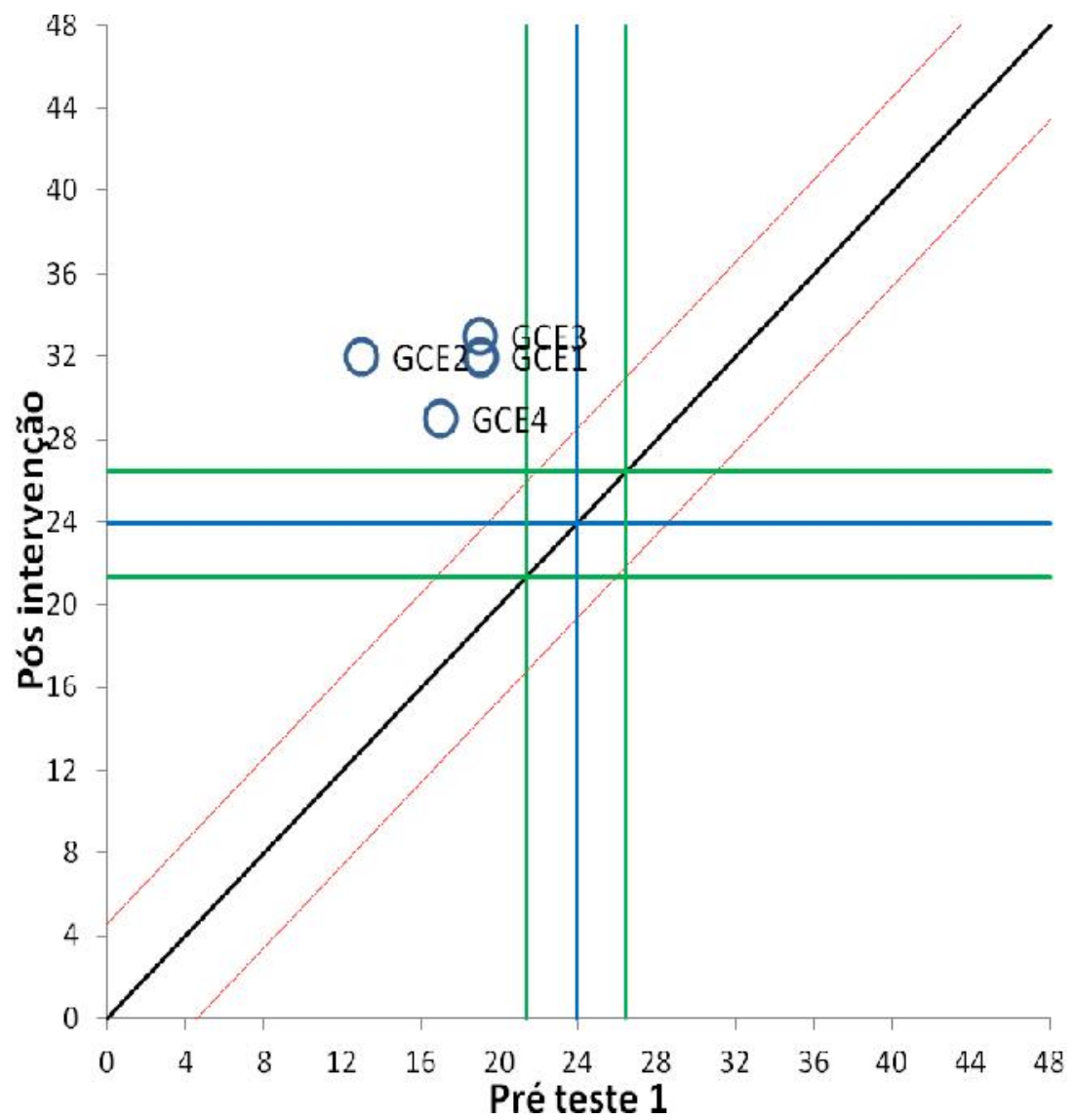

Figura 3 - Resultados da operacionalização do cálculo do IMC e da SC (Critério A) considerando o Escore Geral da Prova de Consciência Fonológica no Grupo Controle de Espera nas situações de pré-teste 1 e pós-teste.

Os resultados apresentados nas Figuras 2 e 3 podem ser considerados evidências da efetividade do programa de intervenção realizado, bem como as potencialidades do Método JT para essa investigação.

\section{Discussão}

Conforme resultados descritos por Sás (2009), após a aplicação da intervenção por meio do programa de remediação fonológica, todos os participantes dos grupos experimental e controle de espera, ainda que não tenham obtido os $100 \%$ de acertos possíveis, obtiveram maior número de acertos em todos os 10 subtestes que constituem a prova. Tais resultados são importantes para o desenvolvimento das habilidades de leitura e escrita, pois como afirma Cardoso-Martins e Frith (1999), a consciência fonológica é um dos mais impor- 
tantes requisitos para a facilidade na aquisição da leitura, sendo a causa e também a condição necessária, porém não suficiente, para o processo.

No estudo atual, o desenvolvimento dos alunos nas habilidades de consciência fonológica foi positivo uma vez que considerando os dados gerais, após a análise dos resultados da intervenção por meio do Método JT, verifica-se que houve melhora após a intervenção para os participantes do estudo no que se refere à Prova de Consciência Fonológica. O Método JT permitiu avaliar os participantes individualmente, diferentemente dos testes estatísticos tradicionais, como o teste "Wilcoxon"2 que permitiria apenas análise geral do grupo. Del Prette e Del Prette (2008), já relatavam que o Método JT atende à necessidade de se investigar as possíveis melhoras de participantes específicos, além da análise das médias grupais em diferentes indicadores de resultados para se verificar a adequação diferencial de determinados procedimentos a características particulares do cliente. Nesse sentido, quanto às mudanças positivas confiáveis para o grupo GE, considerando que GE4 não apresentou mudança confiável após a intervenção, discute-se a possibilidade desse resultado relacionar-se ao fato de que esse participante já apresentava escore relativamente melhor do que os demais participantes antes do início da intervenção. O resultado de GE4, contudo não anula a evidente existência de mudança positiva confiável (MPC) para os participantes GE1, GE2 e GE3 e significância clínica para GE1 e GE2.

Já no GCE, é interessante verificar que antes da intervenção ocorrer, na avaliação obtida no pré-teste 1 e pré-teste 2 (Figura 2), há ausência de mudança de todos os participantes do grupo. Já após a intervenção, na avaliação obtida entre o pré-teste 1 e o pós-teste, observa-se que todos os participantes apresentaram mudanças positivas confiáveis e ainda significância clínica, apoiando a hipótese de que as mudanças realmente ocorreram após a intervenção e que, ainda, no caso da avaliação do pós-teste, ela foi clinicamente significativa. Esse resultado indica ainda outra potencialidade do Método JT a ser discutida, que é a complementaridade deste procedimento de análise também com os delineamentos de linha de base, no caso de estudos usando GE e GCE. Com isso, similarmente ao que ocorre com a estatística inferencial, nestes casos, o Método JT permite: (a) reforçar os resultados observados com o delineamento de linha de base, a partir do cálculo do IMC (possibilidade ou não de atribuir os resultados à intervenção - validade interna); $\mathrm{e}$, (b) investigar também o quanto esses resultados foram efetivos na vida do indivíduo (validade externa).

Os resultados apresentados, corroborando com o estudo de Aguiar et. al (2010), reforçam que o Método JT além de evitar falsos positivos ou negativos de mudanças localizadas dentro da faixa de incerteza de mudança confiável, permite verificar a significância clínica dessas mudanças em termos de recuperação, quando se considera os intervalos de confiança dos pontos de corte que separam a população clínica da não-clínica. Distingue ainda, os participantes que usufruíram positivamente da intervenção e que apresentaram melhora, po- 
Roberta Moreno Sás - Danila Secolim Coser - Miriam Bratfisch Villa Adriana Augusto R. de Aguiar - Maria Amélia Almeida

rém ainda estão abaixo do que seria esperado para o padrão normativo (grupo não-clínico) daqueles que além de melhorarem, podem ser considerados como "recuperados", ou seja, com resultados indicativos do padrão não-clínico. A análise dos resultados evidenciou ainda, na comparação de GE e GCE, que os participantes de GCE apresentaram escores maiores no pós-teste, quanto comparado com GE. Uma hipótese para esses dados pode estar no treino do terapeuta, que já havia aplicado o procedimento de intervenção em participantes anteriores e poderia, portanto, ter desenvolvido uma maior habilidade nas dinâmicas de intervenção propostas. Há também a hipótese de treino prévio dos estudantes com o teste, uma vez que já haviam respondido por duas vezes o mesmo (préteste 1 e 2). Essa última hipótese poderia ser desfeita caso o instrumento fosse utilizado com validação, no qual se indicaria o tempo de espera necessário para uma nova avaliação ou exposição ao mesmo. Além disso, a falta de dados normativos do instrumento de avaliação utilizado no estudo também pode ter influenciado nos resultados da Significância Clínica dos dados.

De acordo com Jacobson e Truax (1992) a falta de familiaridade de muitos profissionais com o uso de indicadores de confiabilidade dos instrumentos; de dados normativos para populações funcional e disfuncional; e/ou de acesso a eles, são limitações para a aplicação do Método em especial para significância clínica. Para avaliação mais precisa da Significância Clínica, seria necessária a produção dos dados normativos da população brasileira para os domínios estudados, a fim de que a significância clínica seja avaliada pelos critérios B e C, como também já demonstra os estudos de Pereira, Silvares e Del Prette (2008) e Yoshida (2008).

Além disso, no caso particular da Educação Especial, outra limitação é a dificuldade de obter a "recuperação" ou retorno à população não-clínica no caso de vários quadros do desenvolvimento atípico, que são objeto desta área de conhecimento (AGUIAR, 2006). Contudo, somente testando proposições teóricas com reais conjuntos de dados, esses problemas podem ser solucionados. Neste estudo, como ocorre com outros estudos na Educação Especial, não só a ausência de dados normativos aplicáveis a esta população justificaria a utilização de uma amostra local como padronização, mas principalmente, o fato de que se trata de uma população de crianças com Sindrome de Down, e que seria improvável haver uma padronização específica para esta população. Assim, justifica-se a utilização da média e desvio padrão referentes à amostra local $(\mathrm{n}=8)$ para cálculo da SC e IMC.

Entende-se, entretanto, que o uso de dados de uma amostra local (obtidos a partir dos próprios participantes do estudo) diminui a confiabilidade dos resultados da análise feita (como ocorre, por exemplo, nas provas estatísticas inferenciais paramétricas/mais fortes em comparação com as nãoparamétricas/menos fortes). Assim, discute-se a importância da comparação dos resultados aqui apresentados com outros estudos visando à replicação desse tipo de análise em conjuntos de dados equivalentes a estes (também usando dados normativos do próprio grupo sob intervenção) e desse mesmo conjunto de 
dados usando valores de validação do próprio instrumento (diante de sua disponibilidade futura).

Como análise final, o Método JT, ao apresentar os dados de mudança confiável tanto para o grupo, como nas distribuições individuais dos escores gerais, mostrou ser uma alternativa muito interessante para apresentação dos dados de maneira clara e objetiva, principalmente se comparada com os dados de porcentagem utilizados de maneira tradicional, que são mais difíceis de serem analisados para amostras pequenas. Nesse aspecto, Aguiar et. al (2010) descreve a utilização deste método como particularmente importante para as pesquisas de intervenção em Educação Especial, pois, em geral, há dificuldade de localizar e de trabalhar com grande número de participantes, o que também impõe limites ao uso de amostras pareadas para delineamento de grupo controle.

Assim como nos estudos de Yoshida (2008), Pereira, Silvares e Del Prette (2008), Sás, Sartori e Almeida (2009) e Aguiar (2006)/ Aguiar et. al (2010), também no presente estudo o Método JT ofereceu vantagens para seu emprego em pesquisas de avaliação de intervenção, e espera-se que este trabalho contribua para divulgar a potencialidade deste método e estimular pesquisadores e profissionais para o seu uso.

\section{Referências}

AGUIAR, A. A. R., DEL PRETTE, Z. A. P., AGUIAR, R. G. \& DEL PRETTE, A. Método JT na EduCação Especial: resultados de um programa de habilidades sociais-comunicativas com deficientes mentais. Educação Especial. v. 23, n. 37, 241-256, 2010.

AGUIAR, A. A. R. de. Construção e avaliação de um programa multimodal de habilidades comunicativas para adultos com deficiência mental. 2006. 202f. Tese (Doutorado em Educação Especial) - Programa de Pós-Graduação em Educação Especial. Universidade Federal de São Carlos, São Carlos, 2006.

AGUIAR, A. A. R.; AGUIAR, R. G.; DEL PRETTE, Z. A. P. Calculando a significância clínica e o índice de mudança confiável em pesquisa-intervenção. 1. ed. São Carlos: EDUFSCar, 2009.

BRASIL, Instituto Brasileiro de Geografia e Estatística (IBGE). Contagem da População 2007. Rio de Janeiro, 2007.

BRYANT, P. E.; BRADLEY, L. Problemas de aprendizagem de leitura. Porto Alegre: Artes Médicas, 1987.

CAPELLINI, S. A. Eficácia do programa de remediação fonológica em escolares com distúrbio específico de leitura e distúrbio de aprendizagem. 226 p. Tese (Doutorado em Ciências médicas), Universidade Estadual de Campinas, Campinas, 2001. 
Roberta Moreno Sás - Danila Secolim Coser - Miriam Bratfisch Villa Adriana Augusto R. de Aguiar - Maria Amélia Almeida

CAPELLINI, S. A.; SALGADO, C. A. Avaliação fonoaudiológica do distúrbio específico de leitura e distúrbio de aprendizagem: critérios diagnósticos, diagnóstico diferencial e manifestações clínicas. In: CIASCA, S. M. (Org.). Distúrbio de aprendizagem: proposta de avaliação interdisciplinar. São Paulo: Casa do Psicólogo, 2003.

CAPELLINI, S. A.; PADULA, N. A.; CIASCA, S. M. Performance of scholars with specific reading disabilities in a remediation program. Pró-Fono, São Paulo, v. 16, n. 3, p. 261-274, 2004.

CAPOVILLA, A. G. S.; CAPOVILLA, F. C. Problemas de leitura e escrita. 2. ed. São Paulo: Memnon Edições Científicas, 2000.

CAPOVILLA, A. G. S.; CAPOVILLA, F. C. Problemas de leitura e escrita: como identificar, prevenir e remediar numa abordagem fônica. 4. ed. São Paulo: Memnon Edições Científicas, 2004.

CARDOSO-MARTINS, C.; FRITH, U. Consciência fonológica e habilidade de leitura na Síndrome de Down. Psicologia: Reflexo Crítico, Porto Alegre, v. 12, p. 209-223, 1999.

COZBY, P. C. Métodos de pesquisa em ciências do comportamento. 1. ed. São Paulo: Atlas. 2003.

DEL PRETTE, Z. A. P.; DEL PRETTE, A. Significância clínica e mudança confiável na avaliação de intervenções psicológicas. Psic.: Teor. e Pesq., Brasília, v. 24, n. 4, p. 497-505, 2008. Disponível em: <http://www.scielo.br/ >. Acesso em: 17 jun. 2009.

FERREIRO, E.; TEBEROSKY, A. Psicogênese da língua escrita. Porto Alegre: Artes Médicas, 1995.

GONZALEZ, O. M. R.; ESPINEL, A. I. G.; ROSQUETE, R. G. Remedial interventions for children with reading disabilities: Speech perception - an effective component in phonological training? Journal of Learning Disabilities, v. 35, n. 4, p. 334-342, 2002.

JACOBSON, N. S.; FOLLETTE, W. C.; REVENSTORF, D. Psychotherapy outcomes research: methods for reporting variability and evaluating clinical significance. Behavior Therapy, v. 15, p. 336-352, 1984.

JACOBSON, N. S; TRUAX, P. Clinical significance: a statistical approach to defining meaningful change in psychotherapy research. In: KAZDIN, A. E. (Ed.), Methodological issues and strategies in clinical research. Washington, 1992. p. 521-538.

Clinical significance: a statistical approach to defining meaningful change in psychotherapy research. Journal of Consulting and Clinical Psychology, v. 59, n. 1, p.12-19, 1991. 
PEREIRA, R. F.; SILVARES, E. F. M.I; DEL PRETTE, Z. A. P. Problemas de comportamento em enuréticos: análise do índice de mudança confiável (IMC). Arquivos Brasileiros de Psicologia, v. 60, n. 3, p.129-141, 2008.

SALGADO, C. A. Programa de remediação fonológica em escolares com dislexia do desenvolvimento. 161p. Dissertação (Mestrado em Ciências Médicas) Universidade Estadual de Campinas, Campinas, 2005.

SÁS, R. M. Efeitos de um programa de remediação fonológica nas habilidades de leitura e escrita em alunos com Síndrome de Down. 2009. 133p. Dissertação (Mestrado) - Universidade Federal de São Carlos, São Carlos, 2009.

SÁS, R.M.; SARTORI, J. R.; ALMEIDA, M.A. Intervenção baseada no programa de remedição fonológica em alunos com dificuldades de aprendizagem escolar. In: V Congresso Brasileiro Multidisciplinar de Educação Especial, 2009, Londrina. Anais do V Congresso Brasileiro Multidisciplinar de Educação Especial. Londrina: Uel, 2009.

YOSHIDA, E. M. P. Significância clínica de mudança em processo de psicoterapia psicodinâmica breve. Paidéia (Ribeirão Preto), v. 18, n. 40, p. 305-316. 2008. Disponível em: <http://www.scielo.br/scielo >. Acesso em: 15 jun. 2010.

Notas

1 Para uma revisão sobre os critérios para cálculo de pontos de corte para significância no Método JT (Critérios A, B e C) ver: Jacobson e Truax (1991); Del Prette e Del Prette (2008); Aguiar, Aguiar e Del Prette (2009).

2 O teste Wilcoxon corresponde a prova estatística não-paramétrica equivalente ao teste "t" para investigação de diferenças entre amostras relacionadas com grupos pequenos.

\section{Correspondência}

Roberta Moreno Sás - Avenida Serafim Gonçalves, 191 CEP:1970-000 - Palmital, São Paulo, Brasil.

E-mail: robertasas@hotmail.com - danilacoser@yahoo.com.br - miriambrat@hotmail.com adrianaaguiar@yahoo.com - ameliama@terra.com.br

Recebido em 17 de outubro de 2011

Aprovado em 09 de janeiro de 2012 